\title{
Ejection Phenomena in Galaxies and QSOs: Are We Going Far Enough?
}

\section{G. Burbidge}

University of California, San Diego, Center for Astrophysics 8 Space Sciences and Department of Physics, La Jolla, CA 92093-0424, U.S.A.

\begin{abstract}
I summarize the observational evidence which suggests: (1) that many of the compact radio sources associated with QSOs and related objects have been ejected from active galaxies so that their redshifts are not measures of their distances, and (2), that the energy emitted by compact sources is not gravitational in origin, but arises through creation processes in regions of galaxies with very strong gravitational fields.
\end{abstract}

I have had the fortune to have lived through all of the early discoveries of the radio sources, both the large radio galaxies and the compact sources. There are still three major issues relating to the extragalactic compact sources, namely:

1. What is the physics of the radiation processes and how good is the theoretical modeling of them?

2. How were the compact sources formed and how did they evolve to their present state?

3. How far away are they, and how do they fit into our view of the universe?

The usual answers to those questions are, first that the radiation is synchrotron and/or Compton radiation. This can account for the spectra all the way from radio waves to gamma rays. However, I suspect that sooner or later we shall have to take seriously the view that coherent plasma radiation may be of importance in understanding the radio flux from some of the rapidly variable sources. The answers to (2) and (3) have been largely driven by the community insisting that in all cases the redshifts of the sources are cosmological in origin, and that the large energies released are gravitational in origin. These two beliefs have led to the conclusion that

(a) the motions seen in many compact sources are superluminal, i.e. compact plasmoids are moving towards us at relativistic speeds such that $\beta=v / c$ lies in the range 0.94 to 0.99 .

(b) gravitational energy is released by the infall of matter into a massive black hole, and is converted into energy that we see at fairly high efficiency $(\approx 10 \%)$.

I believe that there is overwhelming evidence that for many of the radio sources, QSOs and related objects the redshifts $z_{0}$ are composed of two components, $z_{c}$ a cosmological component, and $z_{i}$, an intrinsic component so that $\left(1+z_{o}\right)=\left(1+z_{c}\right)\left(1+z_{i}\right)$. In my oral presentation, I showed much evidence to demonstrate this, statistical evidence that high redshift QSOs and low redshift galaxies are physically associated, pairs of X-ray QSOs ejected from galaxies, also close pairs of QSOs with very different redshifts, etc. For details see Burbidge (1996), Hoyle \& Burbidge (1996), Burbidge, M. (1995, 1997), Arp (1987), 
Burbidge, Hoyle, \& Schneider (1997). Unless all of these results can be shown to be false, radio astronomers must re-evaluate their estimates of sizes, luminosities, superluminal motions, etc. of extragalactic compact sources.

As far as (b) is concerned, serious objections can be raised to the idea that everything we see is a result of the release of gravitational energy. First and foremost as was pointed out by Ambartsumian in the 1960s, we see extensive evidence of ejection from the centers of galaxies - not only of hot gas, relativistic particles, and non-thermal radiation, but also compact galaxies. If we now add to this the evidence just described, namely the association of high redshift QSOs with low redshift galaxies, then we are almost certainly seeing QSOs, andperhaps young galaxies-being ejected from parent systems.

I do not consider that those phenomena can be explained by the black hole accretion disk paradigm. Not only is the efficiency of that process very low if discussed realistically, but it is not able to explain the ejection of coherent massive objects. This needs a new approach to cosmogony involving the creation of mass-energy in galaxies (cf Hoyle \& Burbidge 1996; Hoyle, Burbidge, \& Narlikar 1993, 1994 a,b, 1995).

In summary, I believe that the explosive phenomena which have been discovered through radio astronomy over the last forty years have led us to a new cosmogony in which massive objects in galactic centers lead to creation of new objects, and that this cosmogony is closely tied to an alternative to the popular but misguided hot big bang cosmology. This is the Quasi-steady State Cosmology (Hoyle, Burbidge \& Narlikar, 1993, 1994 a,b 1995).

\section{References}

Arp, H. C. 1987. Quasars, Redshifts 8 Controversies (Berkeley: Interstellar Media).

Burbidge, E. M. 1995. $A \& A, 298$, L1-4.

Burbidge, E. M. 1997. ApJ, 484, L99-101.

Burbidge, G. 1996. A\&A, 309, 9-22.

Burbidge, G., Hoyle, F. \& Schneider, P. 1997. A\&A, 320, 8-12.

Hoyle, F. \& Burbidge, G. 1996. A $B A$, 309, 335-344.

Hoyle, F., Burbidge, G., \& Narlikar, J. V. 1993. ApJ, 410, 437-457.

Hoyle, F., Burbidge, G., \& Narlikar, J. V. 1994a. MNRAS, 267, 1007-1019.

Hoyle, F., Burbidge, G., \& Narlikar, J. V. 1994b. A\&A, 289, 729-739.

Hoyle, F., Burbidge, G., \& Narlikar, J. V. 1995. Proc. Roy. Soc. (London) A, 448, 191-212. 